\title{
Effect of Temperature on the Nanoparticles Agglomerates Fluidization
}

\author{
Ali Asghar Esmailpour \\ School of Chemical Engineering \\ College of Engineering, University of Tehran \\ Tehran, Iran
}

\author{
Reza Zarghami* \\ School of Chemical Engineering \\ College of Engineering, University of Tehran \\ Tehran, Iran \\ *Corresponding author
}

\author{
Navid Mostoufi \\ School of Chemical Engineering \\ College of Engineering, University of Tehran \\ Tehran, Iran
}

\begin{abstract}
The effect of temperature on the fluidization behavior of nanoparticles agglomerates in a fluidized bed was investigated. Moreover, a model was developed based on the force balance between drag, collision, buoyancy and gravitational forces as separation forces and van der Waals force as the attractive force. Results showed that the size of agglomerates decrease by increasing the gas velocity at constant temperature. In addition, the minimum fluidization velocity and the agglomerate size increase with increasing the temperature due to increasing the van der Waals cohesive force. The agglomerate size calculated by the present model is in good agreement with the experimental data.
\end{abstract}

Keywords-nanoparticle; agglomerate; fluidization; force balance; temperature

\section{INTRODUCTION}

Nanoparticles are extensively used in many applications because of their remarkable chemical, physical, optical and electromagnetic properties. Fluidization is one of the most attractive methods of using nanoparticles. Agglomeration is a common phenomenon in nanoparticles fluidization due to cohesive interparticle forces (IPFs) such as van der Waals force.

Two distinct behaviors can be observed in nanoparticles fluidization, termed agglomerate particulate fluidization (APF) and agglomerate bubbling fluidization (ABF) [1-2]. The APF is characterized by smooth fluidization, high bed expansion and uniform distribution of agglomerates throughout the bed [1] while the ABF exhibits large bubbles and the low bed expansion ratio by increasing the gas velocity [1]. Smooth fluidization leads to formation of highly porous agglomerates in the range of several hundred microns

Agglomerates show dynamic behavior during fluidization, breaking and forming again continuously. This can be attributed to the contrast of cohesive forces between nanoparticles and the separation forces originating from the fluid.

Although a number of studies have been carried out to investigate the effect of temperature on particles fluidization
[3-4], none of them has consider the influence of temperature on behavior of nanoparticles agglomerates. Shabanian and Chaouki [3] studied the effect of interparticle force on the gassolid fluidized bed with the help of polymer coating. They controlled the level of interparticle force by temperature and indicated that interparticle forces increased by increasing the temperature. They also found that minimum fluidization velocity increased by enhancing the level of IPFs [3]. Powerful interparticle force in nanoparticles leads to a different behavior compared to other particles. Therefore, the simultaneous effect of temperature, both on the gas and the solid phase, must be considered. Nanoparticle agglomerates have porous and fragile structure and it is difficult to determine their size. Therefore, various models have been proposed for estimating the equilibrium size of agglomerates during fluidization of cohesive powders [5]. Two general approaches are represented in the literature, one of them is based on force balance and the other one is based on energy balance. Chaouki et al. [6] and Morooka et al. [7] were the pioneers of these two categories.

The objective of this study was to investigate the effect of temperature on nanoparticle agglomerates during fluidization. Moreover, a model is developed based on force balance to predict the agglomerate size.

\section{EXPERIMENTAL}

The fluidized bed was a cylindrical column made of glass with an inner diameter of $26 \mathrm{~mm}$ and height of $800 \mathrm{~mm}$. To minimize the humidity of the bed, high-purity and dry nitrogen was used as the fluidizing gas and entered the column through a porous distributor. The gas flow rate was controlled by a mass flow controller (ALICAT model MC-5SLPM). The pressure drop was measured by digital manometer (Dwyer Model 477-000-FM) between two points in the fluidized bed, one located at the top of the column and the other $3.5 \mathrm{~cm}$ above the distributor. The minimum fluidization velocity $\left(u_{m f}\right)$ was experimentally determined by plotting pressure drop vs. superficial gas velocity. All experiments were performed at a temperature range of ambient up to $100{ }^{\circ} \mathrm{C}$. The bed was 
surrounded by two semi cylindrical ceramic heaters, each having $1200 \mathrm{~W}$ electric power.

The powder used in the experiments was hydrophobic (apolar) silica R972 which had primary particles size of $16 \mathrm{~nm}$, primary particles density of $2200 \mathrm{~kg} / \mathrm{m}^{3}$ and bulk density of 85 $\mathrm{kg} / \mathrm{m}^{3}$ (supplied by Evonik industry). Before the experiments, a shaker and a $350 \mu \mathrm{m}$ sieve were used to remove large agglomerates that may have been produced during packing, storage and transportation

The properties of nanoparticle agglomerates were determined by optical microscopy. In order to prevent disrupting of agglomerates, a prudential method was utilized for the sampling. This method involves delicately dipping a rod with a double-sided carbon tape into the fluidized bed and sampling from the surface of the bed. Particular attention was paid during the sampling to avoid breaking the agglomerates.

\section{THEORY}

Nanoparticles become fluidized in the form of agglomerates. To develop a model based on the balance of forces exerted on agglomerates in a fluidized bed, the following assumptions were considered: (1) the agglomerates are spheres of size $d_{a}$; (2) the wall effect is not considered; (3) the van der Waals force is dominant compared to other interparticle forces. Forces acting on agglomerate are drag force $\left(F_{d}\right)$, collision force $\left(F_{c}\right)$, gravitational force $\left(F_{g}\right)$, buoyancy force $\left(F_{b}\right)$ and van der Waals force $\left(F_{v d w}\right)$. Consequently, the force balance equation becomes:

$$
F_{v d w}=F_{d}+F_{c}+F_{b}-F_{g}
$$

One of the common approaches which uses in the nanoparticle fluidization, describes the agglomerates as micron-sized particles with asperities of $0.2 \mu \mathrm{m}$ [6]. This approach believes that the asperity diameter $\left(d_{a s p}\right)$ play main role in van der Waals force:

$$
F_{v d w}=\frac{A_{H} d_{a s p}}{24 z^{2}}
$$

where $A_{H}$ is Hamaker constant and $Z$ is the minimum interparticle distance. The Hamaker constant can be given by:

$$
A_{H}=\frac{3}{4} k_{B} T\left(\frac{\varepsilon_{1}-\varepsilon_{2}}{\varepsilon_{1}+\varepsilon_{2}}\right)^{2}+\frac{3 \hbar v_{e}}{16 \sqrt{2}} \frac{\left(n_{1}^{2}-n_{2}^{2}\right)^{2}}{\left(n_{1}^{2}+n_{2}^{2}\right)^{3 / 2}}
$$

where $T$ is absolute temperature, $K_{B}$ is Boltzman's constant, $h$ is Planck's constant, $v_{e}$ is UV adsorptive frequency, $n_{1}$ is the index of refraction of agglomerates, $\varepsilon_{1}$ is the dielectric constant of agglomerates, $n_{2}$ and $\varepsilon_{2}$ are the index of refraction and dielectric constant of fluid, respectively. It is clear that there is a direct relationship between the Hamaker constant and the temperature.

The main difference between this model and other models is determination of van der Waals force. The agglomerates are formed by sub-agglomerates. The cohesive forces between these sub-agglomerates play an important role in producing the agglomerate [8]. Also, the number of these sub-agglomerates (N) and the porosity of agglomerate should be taken into account. It was assumed that the size of sub-agglomerates $\left(d_{\text {sub }}\right)$ is $35 \mu \mathrm{m}$ [9]. The van der Waals force between two porous subagglomerates can be expressed by [8]:

$$
F_{v d w}=N \frac{A_{H} d_{s u b}\left(\frac{\rho_{\text {sub }}}{\rho_{p}}\right)^{2}}{24 z^{2}}
$$

where $\rho_{\text {sub }}$ is sub-agglomerate density and $\rho_{p}$ is primary particle density. The number of sub-agglomerates in an agglomerate can be determined by the ratio of agglomerate volume $\left(V_{a}\right)$ to sub-agglomerate volume $\left(V_{\text {sub }}\right)$ :

$$
N=\frac{V_{a}\left(1-\varepsilon_{a}\right)}{V_{\text {sub }}}=\left(\frac{d_{a}}{d_{\text {sub }}}\right)^{3}\left(1-\varepsilon_{a}\right)
$$

here, $\varepsilon_{a}$ denotes the agglomerate voidage. It should be noted that the Hamaker constant is a function of temperature and increases with increasing the temperature. Moreover, the temperature affects the density and the viscosity of the gas phase. By substituting the van der Waals force and other forces in Eq. (1), the size of agglomerate can be calculated. Indeed, this equation is a non-linear equation with respect to agglomerate diameter. By selecting the suitable method, the agglomerate size can be obtained.

\section{RESULTS AND DiSCUSSION}

\section{A. Minimum Fluidization Velocity}

Minimum fluidization velocity $\left(u_{m f}\right)$ was obtained using the pressure drop vs. gas velocity $\left(v_{g}\right)$ curve. Pressure drop increases with gas velocity until it reaches to plateau and remains constant. This velocity is called minimum fluidization velocity. The minimum fluidization velocities were analyzed as a first indication of the quality of fluidization.

Figure 1 shows the minimum fluidization velocity of the hydrophobic silica against the temperature. It can be seen in this figure that the minimum fluidization velocity increases with increasing the temperature. This demonstrates that the fluidization quality decreases with increasing temperature. In fact, by increasing the temperature, the APF fluidization tends to the ABF fluidization for hydrophobic silica.

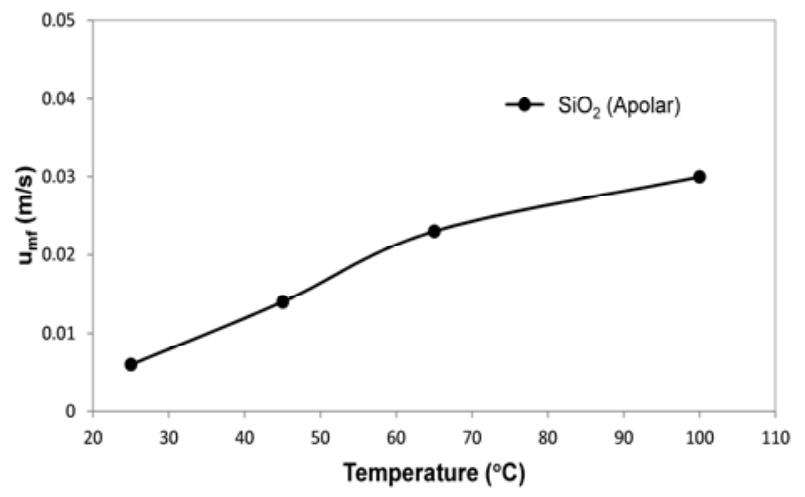

FIGURE I. MINIMUM FLUIDIZATION VELOCITY AT VARIOUS TEMPERATURE 
Tahmasebpoor et al. [10] indicated that increase in minimum fluidization velocity can be due to formation of larger agglomerates caused by an increase of the van der Waals force. The Hamaker constant is directly related to temperature [11]. Therefore, by increasing the temperature, cohesive forces increase and leads to formation of larger agglomerates.

\section{B. Agglomerate Size}

Figure 2 illustrates the variation of estimated agglomerate diameter as a function of the gas velocity at four temperatures. This figure reveals that the size of agglomerates decreases by increasing the gas velocity regardless of the temperature. This can be attributed to the change in the forces related to the gas velocity. By increasing the gas velocity, the drag force and collision force increase. On the other hand, the van der Waals force is a weak function of gas velocity. Therefore, the increment in drag force and collision force as separation forces results in reduction of the agglomerate size.

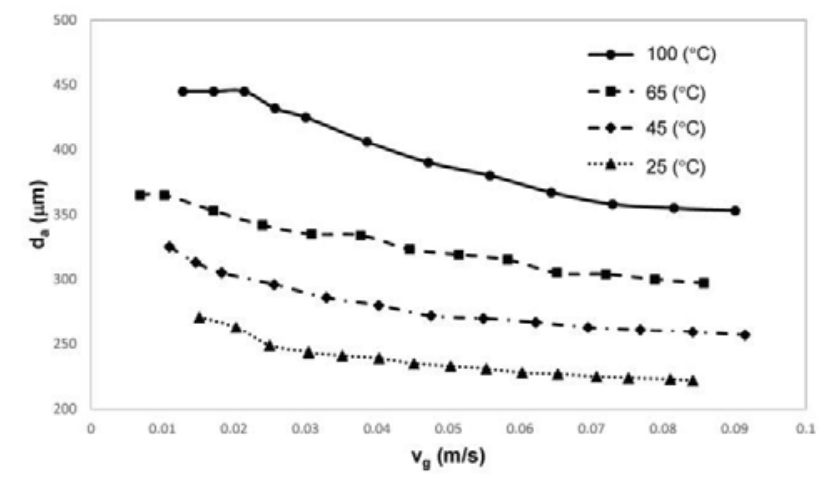

FIGURE II. EFFECT OF GAS VELOCITY ON AGGLOMERATE SIZE

The agglomerate size as a function of temperature at gas velocity of $0.06 \mathrm{~m} / \mathrm{s}$ is shown in Figure 3. It can be seen in this figure that the size of agglomerates increases with increasing the temperature. As explained earlier, by increasing the temperature, the van der Waals force increases significantly. Although the drag force increases with increasing temperature due to changing of gas phase properties, but this cannot overcome the increase in the van der Waals force. In fact, the van der Waals force is a stronger function of temperature than the drag force.

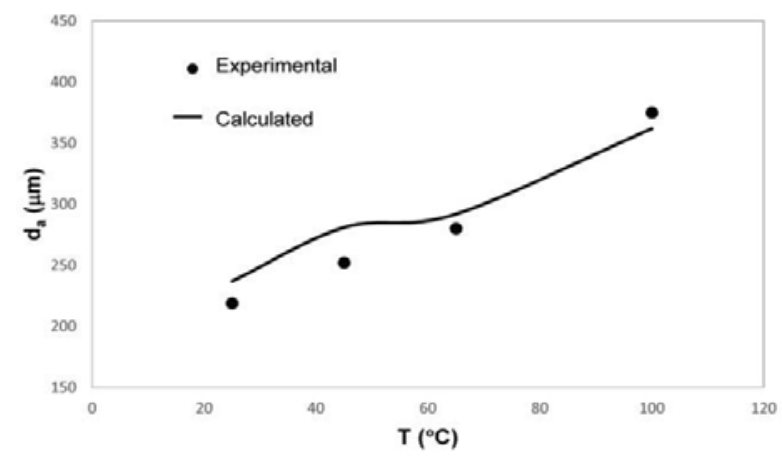

FIGURE III. EFFECT OF TEMPERATURE ON AGGLOMERATE SIZE AT GAS VELOCITY OF 0.06 (M/S)

Figure 3 also presents a comparison between the experimental data and estimated agglomerates size by the model presented on this work at gas velocity of $0.06 \mathrm{~m} / \mathrm{s}$. It can be seen in this figure that a good agreement exists between experimental and calculated values with $7 \%$ average relative error.

Two samples optical microscope images of hydrophobic silica at two temperatures are shown in Figure 4. This Figure also indicated that the agglomerate size increases by increasing the temperature and proved the earlier results.

\section{CONCLUSION}

The effect of temperature on the fluidization behavior of nanoparticle agglomerates was studied experimentally. In addition, a model was developed to determine the size of agglomerates based on the force balance method. It was shown that the minimum fluidization velocity and the agglomerate size increases with increasing the temperature. Using the model, it was also demonstrated that the agglomerate size decreases with increasing the gas velocity at each temperature due to increase in separation forces such as drag and collision forces. The mean agglomerate sizes calculated by the present model are in good agreement with the experimental values.

(A)

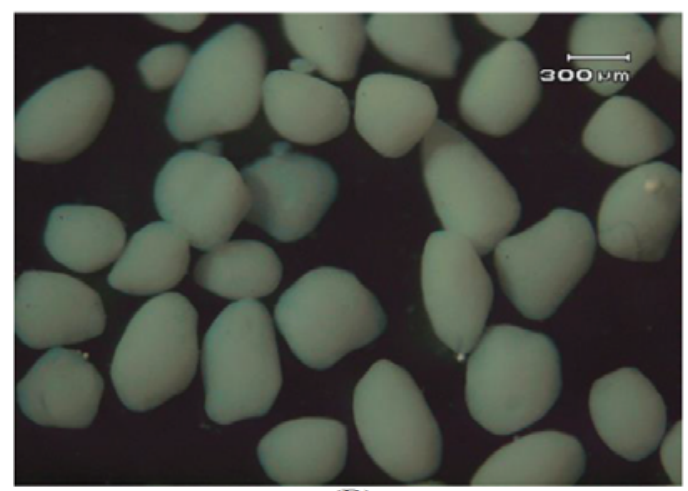

(B)

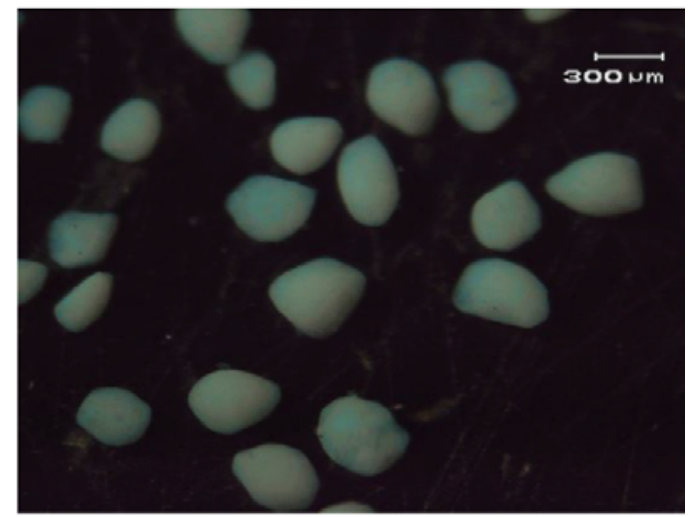

FIGURE IV. OPTICAL MICROSCOPE IMAGE OF HYDROPHOBIC SILICA: (A) $100{ }^{\circ} \mathrm{C}$ (B) $45^{\circ} \mathrm{C}$

\section{REFERENCES}

[1] W. Yao, G. Guangsheng, W. Fei, W. Jun, "Fluidization and agglomerate structure of SiO2 nanoparticles,” Powder Technol., vol. 124, pp. 152-159, 2002.

[2] H. Wang, T. Zhou, J.S. Yang, J.J. Wang, H. Kage, Y. Mawatari, “Model for calculation of agglomerate sizes of nanoparticles in a vibro-fluidized 
bed,” Chem. Eng. Technol., vol. 33, pp. 388-394, 2010.

[3] J. Shabanian, J. Chaouki, "Hydrodynamics of a gas-solid fluidized bed with thermally induced interparticle forces,” Chem. Eng. J., vol. 259, pp. 135-152, 2015.

[4] Q. Guo, J. Zhang, J. Hao, "Flow characteristics in an acoustic bubbling fluidized bed at high temperature,” Chem. Eng. Proc., vol. 50, pp. 331337, 2011.

[5] A. Esmailpour, R. Zarghami, N. Mostoufi, “An Improved Model for Determining Fractal Structure of Nano-Agglomerates,” Can. J. Chem. Eng., in press.

[6] J. Chaouki, C. Chavarie, D. Klvana, "Effect of Interparticle forces on the hydrodynamic behavior of fluidized aerogels,” Powder Technol. Vol. 43, pp. 117-125, 1985.

[7] S. Morooka, K. Kusakabe, A. Kobata, Y. Kato, "Fluidization state of ultrafine powders,” J. Chem. Eng. Jpn. Vol. 21, pp. 41-46, 1988.

[8] L. de Martin, A. Fabre, J.R. van Ommen, "The fractal scaling of fluidized nanoparticle agglomerates,” Chem. Eng. Sci., vol. 112, pp. 7986, 2014.

[9] J.M. Valverde and A. Castellanous, "Fluidization of nanoparticles: A modified Richardson-Zaki law,” AIChE J., vol. 52, pp. 838-842, 2006.

[10] M. Tahmasebpoor, L. de Martin, M. Talebi, N. Mostoufi, J.R. van Ommen, "The role of the hydrogen bond in dense nanoparticle-gas suspensions,” Phys. Chem. Chem. Phys. vol. 15, pp. 5788-5793, 2013.

[11] J.N. Israelachvili, “Intermolecular and Surface Forces," $2^{\text {nd }}$ ed, Academic Press, London, 1992. 